\title{
Family history of cancer and head and neck cancer survival
}

Kayla R. Getz, $\mathrm{MPH}^{1}$, Laura S. Rozek, $\mathrm{PhD}^{2,3}$, Lisa Peterson, $\mathrm{MPH}^{3}$, Emily Bellile, $\mathrm{MS}^{4}$, Jeremy M. G. Taylor, $\mathrm{PhD}^{4}$, Gregory T. Wolf, $\mathrm{MD}^{3}$, Alison M. Mondul, $\mathrm{PhD}^{1}$

1: Department of Epidemiology, University of Michigan School of Public Health, Ann Arbor, Ml

2: Department of Environmental Health Sciences, University of Michigan School of Public Health, Ann Arbor, MI

3: Department of Otolaryngology-Head and Neck Surgery, University of Michigan, Ann Arbor, MI

4: Department of Biostatistics, University of Michigan School of Public Health, Ann Arbor, MI

Running title: Family history and HNSCC survival
Research support was provided by NIH NCI P50 CA097248.

The authors have no conflicts of interest to disclose.

Corresponding author:

Alison M. Mondul, PhD

Department of Epidemiology

University of Michigan School of Public Health

4646 SPH Tower

1415 Washington Heights

Ann Arbor, Michigan 48109-2029

Tel: 734-764-3834

Fax: 734-764-3192

Email: amondul@umich.edu

This is the author manuscript accepted for publication and has undergone full peer review but has not been through the copyediting, typesetting, pagination and proofreading process, which may lead to differences between this version and the Version record. Please cite this article as doi:10.1002/ lary.26524. 


\section{ABSTRACT}

Objective: Patients with a family history of cancer may be genetically predisposed to carcinogenesis. This could affect risk of recurrence, second primary tumors and overall outcomes after treatment of a primary cancer. We evaluated the association between family history of cancer and disease-specific survival in a cohort of patients with primary head and neck squamous carcinoma.

Study Design: 643 incident head and neck squamous carcinoma patients recruited through the University of Michigan Specialized Program of Research Excellence were followed for up to 5 years for survival. Participants were interviewed about personal and family cancer history, demographic information and behavioral habits.

Methods: Cox proportional hazards models were used to estimate the association between family history of cancer in a first degree relative and disease-specific survival.

Results: After multivariable adjustment, we found a non-significant inverse association between family history and HNSCC mortality $(\mathrm{HR}=0.88,95 \% \mathrm{Cl} 0.57-1.35)$. This association was stronger and statistically significant among patients who currently both drank alcohol and smoked cigarettes at diagnosis $(\mathrm{HR}=0.46,95 \% \mathrm{Cl}=0.22-0.97)$; no association was observed among participants who did not both drink and smoke at the time of diagnosis $(\mathrm{HR}=1.14,95 \% \mathrm{Cl}=0.68-1.91 ; \mathrm{p}$-interaction=0.046).

Conclusion: Results from this study suggest that having a family history of cancer may be associated with improved disease-specific survival in patients who use tobacco and alcohol. Additional large studies, particularly in populations including non-whites and 
women, are needed to confirm or refute the association and to elucidate the genetic factors that may underlie this potential association.

Keywords: head and neck cancer, survival, family history, mortality, epidemiology

\section{Level of Evidence: $2 b$}

Abbreviations: HNC-Head and Neck Cancer, ACE- Adult Comorbidity Evaluation, SPORE- Specialized Program of Research Excellence

John Wiley \& Sons

This article is protected by copyright. All rights reserved. 


\section{INTRODUCTION}

Family history is one of the most widely accepted risk factors for development of cancer. For most cancer sites, individuals with a family history of cancer tend to have a 2-3 times greater risk of developing cancer than those without a family history. ${ }^{1}$ Several studies have examined the relationship between family history of cancer and risk of developing a head and neck squamous carcinoma (HNSCC). One large pooled analysis found that individuals with a family history of HNSCC were almost twice as likely to develop the disease as those with no family history of HNSCC, but that there was little association between family history of other types of cancer and risk of developing HNSCC. ${ }^{2}$ Despite this strong evidence for a relationship between family history and HNSCC incidence, there remains little evidence for an association between family history and survival among patients diagnosed with HNSCC.

Survival after diagnosis with HNSCC varies depending on many factors including a patient's behaviors, health status, tumor characteristics, and treatment; it is wellestablished that HPV infection and lower stage at diagnosis have been associated with better HNSCC survival, while tobacco and alcohol use have been associated with poorer outcomes. ${ }^{3}$ This may change somewhat in the future as new staging guidelines which consider HPV status when staging HNSCC patients become widely used. ${ }^{4}$ Patients with an index HNSCC have a well-known high incidence of second primary tumors approaching $4 \%$ per year of follow up that could have a negative influence on overall survival. ${ }^{5}$ Other factors have been examined in relation to survival with less consensus about their association. ${ }^{6}$ One study found that patients with a pessimistic outlook about their cancer had decreased survival compared to optimistic patients who 
were living in a supportive household. ${ }^{7}$ Health insurance status also predicted survival in HNSCC patients; patients who were insured through Medicaid or those who were uninsured had poorer survival than those with other insurance. ${ }^{8}$ However, to our knowledge, no studies have examined the association between a family or personal history of cancer and HNSCC-specific survival.

In the present analysis, we address this gap in the literature by examining the association between family history of cancer and HNSCC-specific survival in a large cohort HNSCC patients recruited through the University of Michigan Head and Neck Cancer Specialized Program of Research Excellence (SPORE).

\section{MATERIALS AND METHODS}

\section{Study Population}

This analysis included 687 participants with incident head and neck cancer recruited through the University of Michigan Head and Neck Cancer SPORE Program. Participants were included if they were diagnosed with previously untreated squamous cell carcinoma of the head and neck from 2008-2013. Patients were recruited from two main clinics that cared for HNSCC patients within the University of Michigan Health System with the approval of the University of Michigan IRB. Participants were not recruited if they had been previously treated for their current HNC, if their HNC was not a primary site but a metastasis from another site, or if they were experiencing recurrent disease from a primary HNSCC diagnosed fewer than five years ago. After excluding participants with missing information about family history of cancer, 643 participants 
were included in the analytic cohort. Sites of HNSCC among the study population were larynx (19.8\%), oropharynx (39.0\%), oral cavity $(37.8 \%)$ and hypopharynx $(3.4 \%)$. There were 473 males and 170 females, ranging in age from 25-95 years old at diagnosis.

Exposure, outcome, and covariate assessment

After diagnosis, each participant was approached by a trained interviewer and completed an initial evaluation. This evaluation collected information about their personal and family history of cancer, demographic information, and behavioral habits. Participants were followed for the next five years; updated exposures were assessed through yearly self-administered surveys that evaluated each participant's physical and emotional well-being, behavioral habits and overall health.

As described above, the main exposure, family history of cancer, was ascertained through the initial evaluation that patients completed at recruitment. Participants were asked if any of their family members had ever been diagnosed with cancer. If the patient responded "yes", the interviewer inquired about the family member's relationship to the patient and what type of cancer they experienced. The interviewer asked the patient if the cancer spread to other sites and if so, where was the first diagnosis to ensure that the patient reported the primary cancer location.

Participants were categorized as having had a family history of cancer if they reported a first degree relative (i.e. a parent, sibling, or child) having had any cancer other than non-melanoma skin cancer. Otherwise, they were classified as not having had a family history of cancer. Sensitivity analyses were conducted examining family history in a parent and family history in a sibling separately. 
Overall survival was determined through clinical patient follow up, medical records of treating or referring physicians, annual surveys or personal communication with family members. Linkage with the Social Security Death Master File (SSDMF) was conducted annually to ascertain participant survival if patients were lost to follow up. If a participant was in the SSDMF, did not respond to the mailed survey, or if a family member informed us that they had died, medical records were reviewed by trained study personnel to determine the date of death and whether the death was due to HNSCC or another cause. Follow-up time was calculated from the date of diagnosis to date of death from HNSCC, death from another cause, or the censoring date (2/1/2014), whichever was earliest. The median survival time was 24.4 months. Cases were defined as death from HNSCC as the underlying cause $(n=95)$; patients with all other causes of death were censored at their death date.

\section{Statistical Analysis}

Cox proportional hazards models were used to estimate the hazard ratio (HR) and $95 \%$ confidence interval $(\mathrm{Cl})$ of death from HNSCC according to family history of cancer (yes/no). The following factors known or hypothesized to be associated with risk of HNSCC or to influence cancer survival were included as potential confounders. Age at diagnosis, tobacco and alcohol use were gathered from the initial evaluation at baseline. HPV status was assessed by PCR technique for patients whose tumor tissue was available and was assigned positive, negative or missing for each participant based on test results. Comorbidities were measured by utilizing Adult Comorbidity Evaluation scale, a validated medical record-based comorbidity instrument, and each participant 
was assigned a score $(0=$ No comorbidities, $1=$ Mild, $2=$ Moderate, $3=$ Severe $)$ based on comorbidities recorded in the patient's medical record. ${ }^{9}$ In addition, tumor-specific factors known to be associated with survival such as primary tumor site and tumor stage of each individual's cancer were also included in the model. Thus, our final multivariable model included the following variables: smoking status (current, former, never), drinking status (current, former, never), ACE score (none, mild or moderate/severe), stage $(1,2$, 3, or 4), site (larynx, oral cavity, oropharynx, or hypopharynx), personal history of HNC (yes or no), and HPV status (negative, positive or missing). The association between family history and the hazard of death from HNSCC was also estimated for the following subsets of patients: age at diagnosis $\leq 50$ vs $>50$ years, current drinkers and smokers vs. all others, HPV positive vs. negative, and disease site of hypopharynx and larynx vs. oral cavity and oropharynx. Statistical interaction was assessed using the likelihood ratio test. All analyses were conducted using SAS version 9.4.

\section{RESULTS}

Characteristics of the participants by family history of cancer are shown in Table 1. Participants with a family history of cancer were more likely to be former $(35.5 \%)$ or never (24.5\%) smokers and participants without a family history were more likely to currently smoke (46.8\%). Participants with a family history were more likely to be former drinkers compared to current drinkers, but the proportion of never drinkers in both groups was the same. The distribution of primary tumor sites of head and neck cancer were similar between groups but among participants with a family history of cancer the most common site was oropharynx, whereas among participants without a family history 
of cancer, the most common site was oral cavity. The stage distribution at diagnosis with HNSCC differed by family history of cancer; patients with a family history of cancer were slightly more likely to be diagnosed at stage 1 , whereas those without a family history were slightly more likely to be diagnosed at stage 4 . Patients with a family history of cancer were more likely to have a personal history of cancer and were more likely to have moderate comorbidities compared to the group of patients without a family history of cancer (Table 1).

After adjustment for age at diagnosis, we observed a non-significant inverse association between family history of cancer and death in HNSCC patients (HR=0.83, $95 \% \mathrm{Cl}=0.55-1.26, p=0.38)$. Further adjustment for several potential confounding variables did not meaningfully change the association $(\mathrm{HR}=0.88,95 \% \mathrm{Cl} 0.57-1.35$, $p=0.56$ ) (Table 2). There was no difference in the association when we looked at family history in a parent or a sibling separately (data not shown). We were unable to examine family history of HNSCC separately; only 22 participants had a family history of HNSCC and only 3 of them died of HNSCC during follow-up.

We observed an interaction with tobacco and alcohol use such that among participants who currently both drank alcohol and smoked cigarettes at the time of diagnosis, a family history of cancer was associated with better survival $(H R=0.46,95 \%$ $\mathrm{Cl}=0.22-0.97)$; no association was observed between family history of cancer and HNC survival among participants who did not both drink and smoke at the time of diagnosis $(\mathrm{HR}=1.14,95 \% \mathrm{Cl}=0.68-1.91 ; \mathrm{p}$ for interaction $=0.046$; Table 3$)$. There was an inverse association between family history and mortality in participants without HPV infection and in those whose cancer was in the hypopharynx or larynx, but these 
interactions were not statistically significant (Table 3). There was no interaction by age at diagnosis (Table 3). Further adjustment for stage in any subgroup did not meaningfully alter the findings (data not shown).

\section{DISCUSSION}

In this study of HNSCC patients, although a family history of cancer was not significantly associated with survival overall, it was associated with improved survival in HNSCC patients who were currently drinking and smoking at the time of diagnosis. A large, pooled analysis of family history and HNC incidence found increased risk of developing $\mathrm{HNC}$ in the same subgroups, providing coherent evidence that family history may be related to HNC due to tobacco and alcohol exposure. ${ }^{2}$ To our knowledge, this is the first study to examine family history of cancer in relation to survival among HNC patients. However, this relationship has been studied in other cancers.

Several studies have investigated how a family history of colorectal cancer is related to survival among colorectal cancer patients. A study by Lee et al. found that patients with stage 3 colon cancer who had a family history had better overall and disease free survival than patients without a family history of colorectal cancer. ${ }^{10}$ In another study of patients with colorectal cancer; Morris et al. found similar results. Patients who had a family history of colon cancer had better survival than those without a family history of colon cancer and they also observed that patients who had more than one relative with a history of colon cancer had even better survival than those without a family history of cancer. ${ }^{11}$ Similar results have been reported about the relationship with survival among breast cancer patients who have a family history of breast cancer. A study by Malone et al. reported that, among women under the age of 45 with invasive 
breast cancer, those who had a family history had better survival. ${ }^{12}$ Russo et al. reported that breast cancer cases with a first degree relative with a history of breast cancer had a non-statistically significant better survival than cases without a family history.$^{13}$ It should be noted that the family history-survival association may differ for patients with an inherited familial cancer syndrome.

Fewer studies have examined family history of any cancer in relation to survival among patients with cancer at a particular site. A study by Isla et al. investigated the relationship between family history of any cancer and overall survival of women with lung cancer. Lung cancer patients who had a family history of any type of cancer had significantly longer survival than those without a family history of cancer; median overall survival was 23.0 months for lung cancer patients without a family history of cancer and median overall survival for lung cancer patients with a family history of various types of cancer was 25.3 months. ${ }^{14}$ They also determined that participants with a family history of cancer had a significantly higher proportion of current smokers compared to those who did not have a family history of cancer. ${ }^{14}$ These findings are similar to those reported in the current analysis.

It is difficult to pinpoint the exact reason why having a family history of cancer may improve survival. That it has been observed across different cancer types with the exception of inherited cancer syndromes suggests that the finding may not be due to chance. One frequently suggested explanation for the findings for colorectal and breast cancer is lead-time bias: i.e. individuals with a family history may undergo more aggressive screening, and ultimately become diagnosed at an earlier stage which would lead to improved survival. ${ }^{13}$ Because screening for HNC is not common, the protective 
association we observed cannot be explained by screening. In addition, adjustment for stage at diagnosis did not alter our results for family history in any subgroup, suggesting that lead-time bias does not explain our findings. It is possible that delay in diagnosis and treatment, regardless of tumor stage could influence survival outcomes ${ }^{15}$ with potentially less delay in patients with a family history, however we did not have such data for this cohort.

To our knowledge, this is the first analysis assessing the relationship between family history of cancer and survival among HNSCC cases. Our study has many strengths including a large sample size representing various sites of HNSCC, excellent survival ascertainment, and information on many clinical tumor characteristics, potential confounders, and effect modifiers. One potential limitation of our study is that family history of cancer was self-reported. It is unlikely that any misclassification of family history would be different in those patients who survived compared to those that did not; thus any misclassification would be non-differential and would bias our findings toward the null. Thus, the true protective association between family history and HNC survival among those exposed to tobacco and alcohol might actually be stronger than what we observed. Our study population consisted mainly of white men, so these results may not be generalizable to other races or to women. Finally, although the differences in demographics in this retrospective analysis were not significant, there was a tendency for patients with a family history to have a lower percentage of severe co-morbidities and higher frequency of oropharyngeal cancers and Stage I tumors which, despite our adjustment for them as potential confounding factors, could influence outcomes.

\section{CONCLUSIONS}


Results from this study suggest that having a family history of cancer may be associated with improved disease-specific survival in HNSCC patients who use tobacco and alcohol. Additional large studies, particularly in populations including non-whites and women, are needed to confirm or refute the suggested association and to elucidate potential genetic factors that may underlie this association before recommendations can be translated in the clinical setting.

\section{Acknowledgements:}

This study would not have been possible without the generosity and participation of our patients and the dedicated assistance and teamwork of the members of the Head and Neck Oncology Program at the University of Michigan who enrolled and cared for the patients in this study. The authors thank the many investigators in the University of Michigan Head and Neck Specialized Program of Research Excellence for their contributions to patient recruitment, assistance in data collection and encouragement including Carol R. Bradford, MD, Thomas E. Carey, PhD, Douglas B. Chepeha, MD, Sonia Duffy, PhD, Avraham Eisbruch, MD, Joseph Helman, DDS, Kelly M. Malloy, MD, Jonathan McHugh, MD, Scott A. McLean, MD, Tamara H. Miller, RN, Jeff Moyer, MD, Mark E. Prince, MD, Nancy Rogers, RN, Matthew E. Spector, MD, Nancy E. Wallace, RN, Heather Walline, PhD, Brent Ward, DDS, and Francis Worden, MD. 


\section{References}

1. Peto J, Houlston RS. Genetics and the common cancers. Eur J Cancer. 2001; 37 Suppl 8: S88-S96.

2. Negri E, Boffetta P, Berthiller J, et al. Family history of cancer: pooled analysis in the International Head and Neck Cancer Epidemiology Consortium. Int J Cancer. 2009; 124(2):394-401.

3. Marur S, D'Souza G, Westra WH, Forastiere AA. HPV-associated head and neck cancer: a virus-related cancer epidemic. Lancet Oncol. 2010; 11(8): 781-789.

4. AJCC. AJCC Cancer Staging Manual. $8^{\text {th }}$ ed. New York: Springer; 2017.

5. Wu X, Spitz MR, Lee JJ, et al. Novel Susceptibility Loci for Second Primary Tumors/Recurrence in Head and Neck Cancer Patients: Large Scale Evaluation of Genetic Variants. Cancer prevention research (Philadelphia, Pa). 2009;2(7): 617-624.

6. Duffy SA, Ronis DL, McLean S, et al. Pretreatment health behaviors predict survival among patients with head and neck squamous cell carcinoma. J Clin Oncol. 2009; 27(12): 1969-1975.

7. Allison PJ, Guichard C, Fung K, Gilain L. Dispositional optimism predicts survival status 1 year after diagnosis in head and neck cancer patients. J Clin Oncol. 2003; 21(3): 543548.

8. Kwok J, Langevin SM, Argiris A, Grandis JR, Gooding WE, Taioli E. The impact of health insurance status on the survival of patients with head and neck cancer. Cancer. 2010; 116(2): 476-485.

9. Piccirillo JF, Tierney RM, Costas I, Grove L, Spitznagel EL. Prognostic importance of comorbidity in a hospital-based cancer registry. JAMA. 2004; 291(20): 2441-2447.

10. Lee SD, Kim BC, Han KS, et al. Influence of family history on survival in patients with colon and rectal cancer. J Dig Dis. 2014; 15(3): 108-115. 
1. Morris EJ, Penegar S, Whitehouse LE, et al. A retrospective observational study of the relationship between family history and survival from colorectal cancer. $\mathrm{Br} J$ Cancer. 2013; 108(7): 1502-1507.

2. Malone KE, Daling JR, Weiss NS, McKnight B, White E, Voigt LF. Family history and survival of young women with invasive breast carcinoma. Cancer. 1996; 78(7): 14171425.

3. Russo A, Herd-Smith A, Gestri D, et al. Does family history influence survival in breast cancer cases? Int J Cancer. 2002; 99(3): 427-430. cancer patients: a population-based study from southern Sweden. J Clin Oncol. 1998; 16(2): 397-404.

4. Isla D, Felip E, Vinolas N, et al. Lung cancer in women with a family history of cancer: The spanish Female-specific Database WORLD07. Anitcancer Research. 2016; 36 (12): 6647-6653.

5. Murphy CT, Galloway TJ, Handorf EA, et al. Survival Impact of Increasing Time to Treatment Initiation for Patients With Head and Neck Cancer in the United States. J Clin Oncol. 2015.

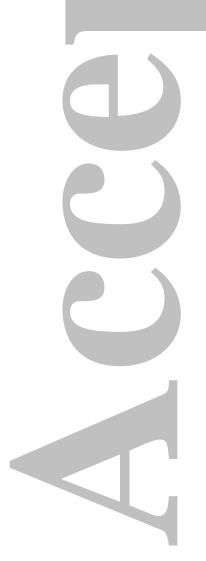


Table 1. Selected baseline characteristics [means (standard deviation) or number (percent)] of SPORE patients by family history of cancer

\begin{tabular}{|c|c|c|c|}
\hline & \multicolumn{2}{|c|}{ Family History } & \multirow[b]{2}{*}{ p-value } \\
\hline & Yes & No & \\
\hline $\mathrm{N}$ & 374 & 269 & \\
\hline Age at Diagnosis (years) & $62.7 \pm 11.4$ & $59.0 \pm 12.2$ & 0.0001 \\
\hline \multicolumn{4}{|l|}{ Sex } \\
\hline Male & $276(73.8 \%)$ & $197(73.2 \%)$ & 0.87 \\
\hline \multicolumn{4}{|l|}{ Race } \\
\hline White & $357(95.5 \%)$ & $253(94.1 \%)$ & 0.43 \\
\hline BMI $\left(\mathrm{kg} / \mathrm{m}^{2}\right)$ & $27.0 \pm 6.1$ & $27.2 \pm 6.1$ & 0.64 \\
\hline \multicolumn{4}{|l|}{ Smoking Status } \\
\hline Current & $150(40.1 \%)$ & $126(46.8 \%)$ & 0.23 \\
\hline Former & $132(35.3 \%)$ & $82(30.5 \%)$ & \\
\hline Never & $92(24.6 \%)$ & $61(22.7 \%)$ & \\
\hline \multicolumn{4}{|l|}{ Drinking Status } \\
\hline Current & $237(63.4 \%)$ & $187(69.5 \%)$ & 0.20 \\
\hline Former & $102(27.3 \%)$ & $57(21.2 \%)$ & \\
\hline Never & $35(9.3 \%)$ & $25(9.3 \%)$ & \\
\hline \multicolumn{4}{|l|}{ Disease site } \\
\hline Larynx & $78(20.9 \%)$ & $49(18.2 \%)$ & 0.47 \\
\hline Oral Cavity & $137(36.6 \%)$ & $114(42.4 \%)$ & \\
\hline Oropharynx & $147(39.3 \%)$ & $96(35.7 \%)$ & \\
\hline Hypopharynx & $12(3.2 \%)$ & $10(3.7 \%)$ & \\
\hline \multicolumn{4}{|l|}{ Stage at diagnosis } \\
\hline $1 \longrightarrow$ & 67 (17.9\%) & $36(13.4 \%)$ & 0.45 \\
\hline 2 & $43(11.5 \%)$ & $30(11.2 \%)$ & \\
\hline 3 & $53(14.2 \%)$ & $38(14.1 \%)$ & \\
\hline 4 & $211(56.4 \%)$ & $165(61.3 \%)$ & \\
\hline \multicolumn{4}{|l|}{ Treatment } \\
\hline Surgery alone & $98(26.2 \%)$ & $59(21.9 \%)$ & 0.42 \\
\hline Surgery + adjuvant & $70(18.7 \%)$ & $61(22.7 \%)$ & \\
\hline Radiation (+/- adjuvant surgery) & $26(7.0 \%)$ & $18(6.7 \%)$ & \\
\hline Chemoradiation ( $+/$ - adjuvant surgery) & $155(41.4 \%)$ & $106(39.4 \%)$ & \\
\hline $\begin{array}{l}\text { Chemotherapy alone or } \\
\text { palliative therapy }\end{array}$ & $25(6.7 \%)$ & $25(9.3 \%)$ & \\
\hline \multicolumn{4}{|l|}{ HPV Status } \\
\hline Positive & $80(38.7 \%)$ & $54(38.8 \%)$ & 0.87 \\
\hline \multicolumn{4}{|l|}{ Personal History of Cancer } \\
\hline Yes & $98(26.2 \%)$ & $42(15.6 \%)$ & 0.001 \\
\hline $\begin{array}{c}\text { Ace Score } \\
\text { None }\end{array}$ & & 77 (28 6\%) & רח \\
\hline $\begin{array}{l}\text { None } \\
\text { Mild }\end{array}$ & $92(24.6 \%)$ & $77(28.6 \%)$ & 0.02 \\
\hline Mild & $168(44.9 \%)$ & $136(50.6 \%)$ & \\
\hline Moderate/Severe & $114(30.5 \%)$ & $56(20.8 \%)$ & \\
\hline
\end{tabular}


Table 2. Association between family history of cancer and HNC mortality among patients in the University of Michigan Head and Neck Cancer SPORE

\begin{tabular}{llcccc}
\hline & & \# HNC Deaths & Person-Years & HR (95\% Cl) & HR (95\% Cl) \\
\hline Family History & No & 42 & 606.33 & $1.0($ ref $)$ & $1.0($ ref $)$ \\
& Yes & 53 & 844.16 & $0.83(0.55,1.26)$ & $0.88(0.57,1.35)$
\end{tabular}

* - Adjusted for Age at Diagnosis

$\dagger$ - Adjusted for Age at Diagnosis, Personal History of Cancer, Personal History of HNC, Disease Site, Stage, ACE Score, Smoking Status, Drinking Status, and HPV Status 
Table 3 - Association* between family history of cancer and HNC mortality among patients in the University of Michigan Head and Neck Cancer SPORE, stratified by selected potential effect modifiers

\begin{tabular}{|c|c|c|c|c|c|c|}
\hline & & \multicolumn{2}{|c|}{ No Family History } & \multicolumn{2}{|c|}{ Yes Family History } & \multirow[b]{2}{*}{$p$ for interaction } \\
\hline & & $\begin{array}{l}\text { \# HNC } \\
\text { Deaths }\end{array}$ & HR (95\% Cl) & $\begin{array}{l}\text { \# HNC } \\
\text { Deaths }\end{array}$ & HR (95\% Cl) & \\
\hline \multirow[t]{2}{*}{ Age at Diagnosis } & $\leq 50$ & 12 & 1.0 (ref) & 30 & $1.18(0.48-2.86)$ & \multirow[b]{2}{*}{0.58} \\
\hline & $>50$ & 9 & 1.0 (ref) & 44 & $0.88(0.54-1.44)$ & \\
\hline \multirow{2}{*}{$\begin{array}{l}\text { Current Drinker } \\
\text { and Smoker }\end{array}$} & No & 22 & 1.0 (ref) & 42 & $1.14(0.68-1.91)$ & \multirow[b]{2}{*}{0.046} \\
\hline & Yes & 20 & 1.0 (ref) & 11 & $0.46(0.22-0.97)$ & \\
\hline \multirow[t]{2}{*}{ HPV status } & Negative & 22 & 1.0 (ref) & 25 & $0.60(0.33-1.08)$ & \multirow[b]{2}{*}{0.47} \\
\hline & positive & 5 & 1.0 (ref) & 7 & $0.96(0.30-3.03)$ & \\
\hline \multirow{2}{*}{\multicolumn{2}{|c|}{ 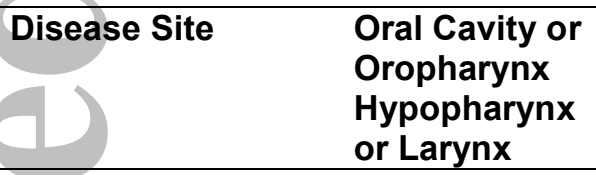 }} & 29 & 1.0 (ref) & 39 & $0.96(0.59-1.55)$ & \multirow[b]{2}{*}{0.23} \\
\hline & & 13 & 1.0 (ref) & 14 & $0.55(0.26-1.18)$ & \\
\hline
\end{tabular}

- Adjusted for Age at Diagnosis

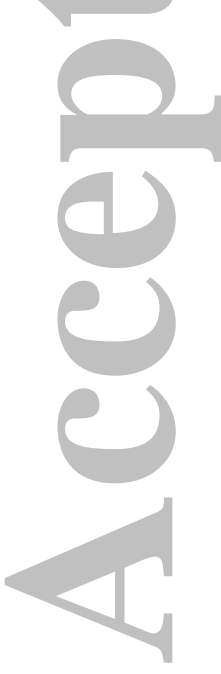

